\title{
Complete characterisation of attosecond SXR pulses generated by MIR laser sources
}

\author{
Thomas Gaumnitz , Arohi Jain, Martin Huppert, Inga Jordan, Fernando Ardana-Lamas and \\ Hans Jakob Wörner
}

ETH Zürich, Laboratory of Physical Chemistry, Vladimir-Prelog-Weg 2, CH-8049 Zürich, Switzerland

\begin{abstract}
Attosecond streaking with broadband SXR continua leads to contributions from multiple overlapping lines in the photoelectron spectrum. The Volkov-transform generalized projection algorithm (VTGPA) is generalised to include all contributing photoelectron bands (multi-line VTGPA) for the reconstruction of ultra-broadband SXR continua. We further investigate the influence of the collection angle of photoelectron detectors on attosecond streaking spectrograms and show full reconstruction for angle-integrated streaking traces. Also, the effects of the photoionization dipole matrix elements on the reconstruction are demonstrated.
\end{abstract}

\section{Introduction}

Attosecond metrology had been limited to Ti:Sa lasers combined with an active stabilization of the carrier-envelope phase (CEP), where the achievable photon energy was limited to $\sim 100 \mathrm{eV}$. The quadratic scaling of the ponderomotive energy with wavelength enables to generate photon energies in the soft-X-ray (SXR) domain with MIR laser sources. This has allowed access to X-ray absorption edges of most second- and third-row elements which are central to chemistry, biology and material science. To obtain the highest temporal resolution in a pump-probe experiment, the attosecond pulses synthesized from a SXR supercontinua and the mid-infrared (mid-IR) pulses have to be carefully characterized.

\section{Methods}

Using long wavelength drivers, the HHG cut-off $\left(\sim \mathrm{I} \lambda^{2}\right)$ can be extended on cost of the HHG flux that scales approximately with $\lambda^{-(5-6)}$. For ultra-broadband SXR continua, helium presents the advantage of a single photoelectron line, at the cost of a low cross section. To mitigate the issues of lower photon flux and cross sections, spectrometers with large collection angle, like magnetic-bottle TOFs (MB-TOF) or VMIs, can be used to collect as many photoelectrons as possible, resulting in streaking spectrograms shown in Fig. 1. Besides the increased photoelectron signal, the shape of the spectrogram changes drastically, as seen for different maximum collection angles in Fig. 1(A)-(C), which has to be taken into account during the reconstruction. Alternatively, target gases other than helium with higher cross

*Corresponding author: Thomas.Gaumnitz@phys.chem.ethz.ch 
sections (cf. Fig. 2A) can be used, but then multiple orbitals are simultaneously ionized leading to overlapping energy shifted streaking-spectrograms as shown in Fig. 3A. However, the reconstruction in both cases requires an extension of the traditional streaking reconstruction formalism which incorporates all overlapping orbitals and takes the effect of the angle-dependent dipole transition amplitudes into account.
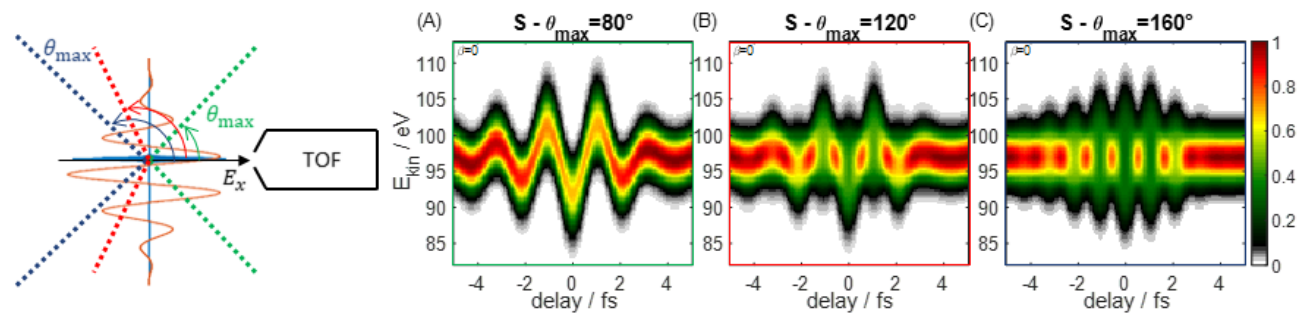

Fig. 1. SXR- and IR-field polarization and the time-of-flight detector together for three polar collection angles of the TOF. Simulated streaking traces $\mathrm{S}(\mathrm{E}, \tau)$ in helium with a 350 as SXR pulse centred at 110 $\mathrm{eV}$ photon energy for collection polar angles of $\theta_{\max }=80^{\circ}(\mathrm{A}), \theta_{\max }=120^{\circ}(\mathrm{B})$, and $\theta_{\max }=160^{\circ}(\mathrm{C})$ normalized on the peak count rate assuming an isotropic photoelectron angular distribution (PAD), corresponding to an anisotropy parameter $\beta=0$.

In Fig. 2B, we present our recent generalization of the VTGPA [3] accounting for multiple overlapping photoelectron bands and angle-integrated streaking spectrograms [4, 5]. This method allows for the complete temporal reconstruction of both IR and SXR pulses by including the accurate target atom complex photoionization matrix elements (PMEs), as Fourier transformation is avoided in the reconstruction routine and therefore overcomes the limitations caused by the central momentum approximation (CMA).

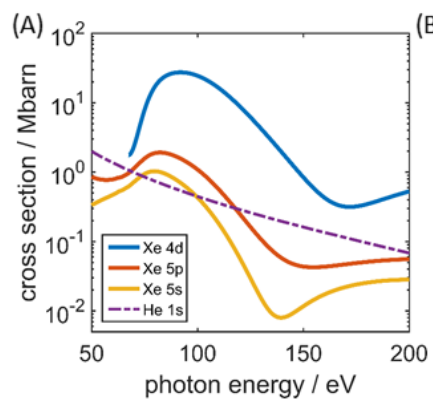

(B) ML-VTGPAincluding PAD

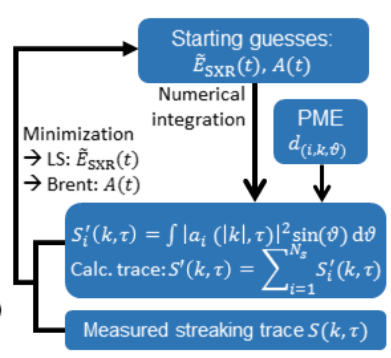

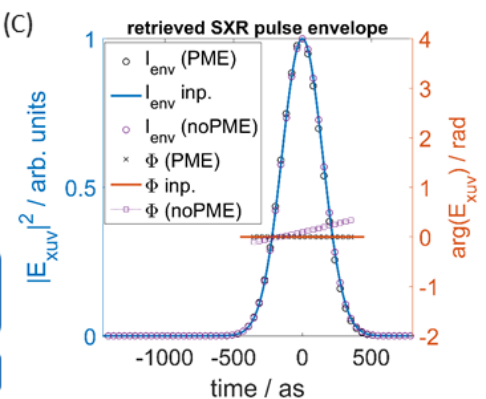

Fig. 2. (A) Photoionization cross sections for xenon $4 d, 5 s$, and $5 p$ photoelectron bands calculated from the complex PMEs [1], including the comparison with the He cross section [2]. (B) Flow chart of the ML-VTGPA method including PAD. Initial guesses for Esxr $(t)$ and the mid-IR vector potential A(t) enter the iterative algorithm which consists of numerical integration of the SFA equation using the

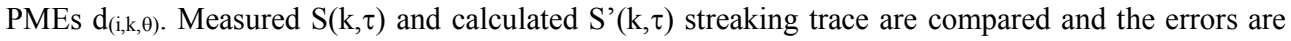
minimized with least-squares and Brent's method for the SXR and mid-IR pulses, before the new guesses are fed into the next iteration of the algorithm. (C) Comparison of retrieved SXR pulse from Fig. 1(B) including (black circles/crosses) and excluding (violet circles/squared) the xenon PMEs.

\section{Results}

In Fig. 3, we have demonstrated numerically that our multi-line VTGPA algorithm is perfectly suitable for the retrieval of chirped SXR pulses and chirped streaking fields in the near- and mid-infrared spectral region. 

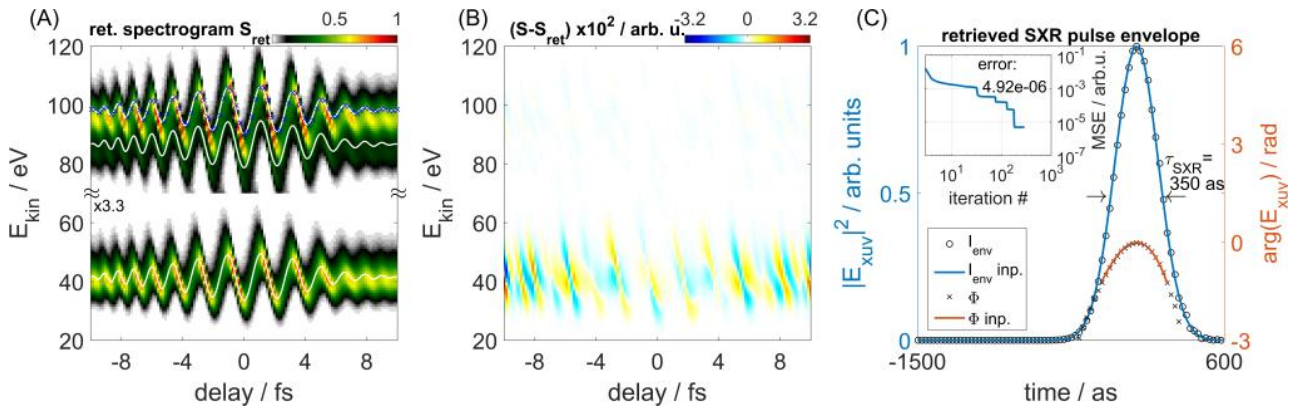

Fig. 3. Reconstructed (A) streaking spectrogram Sret to test the ML-VTGPA algorithm with a chirped attosecond pulse of $\tau_{\mathrm{SXR}}=350$ as in Xe including the PMEs for the $5 \mathrm{p}, 5 \mathrm{~s}$ (both multiplied by 3.3, for better visibility of all photoelectron bands) and $4 \mathrm{~d}$ photoelectron bands. The input vector potential $\mathrm{A}(\mathrm{t})$ - strongly chirped pulse - (white line) and the reconstructed vector potential (blue crosses) are scaled and plotted on top of the $5 \mathrm{p}$ photoelectron line for comparison. (B) False colour representation of the difference between $\mathrm{S}$ and $\mathrm{S}$ ret multiplied by two orders of magnitude. The residual mean square error between input and reconstructed spectrogram was found to be $4.92 \times 10^{-6}$ after $\sim 500$ iterations of the algorithm. (C) Reconstructed SXR pulse parameters amplitude Ienv $(t)$ and phase $\Phi(t)$ (black circles and crosses) for detailed comparison with the input SXR pulse parameters (blue, red lines).

We have used the method described above to study partially angle-integrated streakingspectrograms as obtained from MB-TOF spectrometers, where a complete and accurate reconstruction of attosecond pulses for large collection angles was obtained. The retrieval of the attosecond pulse for $\theta_{\max }=120^{\circ}$ is shown in Fig. 2C. As seen in Fig. 2A, the photoionization cross sections are not flat and the influence of photoemission matrix elements (PMEs) on broadband pulses cannot be ignored. To investigate the effect of the PMEs on the retrieval, we tested the reconstruction algorithm with and without including the PMEs. The amplitude of the input pulse is given in blue together with the retrieved amplitudes including (black circles) and neglecting (violet circles) the PMEs and shows a very good agreement with the input pulse parameters. A discrepancy between the input and the retrieved SXR pulse phase can be observed in Fig. 3C for the reconstruction without PME (violet crosses).

The ML-VTGPA algorithm has been applied to streaking measurements in xenon with multiple overlapping photoemission lines presented in [5] and allowed the reconstruction of an isolated 43-as SXR pulse. The broadband SXR continua covering the energy range between 60 and $180 \mathrm{eV}$ enable the measurement of element- and site-specific X-ray absorption spectra with unprecedented temporal resolution at the L-edges of silicon $(99 \mathrm{eV})$, phosphor $(136 \mathrm{eV})$, and sulfur $(163 \mathrm{eV})$, and therefore expand the scope of attosecond science in the gas and liquid phase.

\section{References}

1. A. S. Kheifets, Phys. Rev. A 87, 063404 (2013).

2. G. V. Marr, J.B. West, At. Data Nucl. Data Tables 18.5, 497-508 (1976).

3. P. D. Keathley, S. Bhardwaj, J. Moses, G. Laurent, F. X. Kärtner, New J. Phys, 18(7), 114 (2016).

4. T. Gaumnitz, A. Jain, H.J. Wörner, Opt. Express, 26(11), 14719 (2018).

5. T. Gaumnitz, A. Jain, Y. Pertot, M. Huppert, I. Jordan, F. Ardana-Lamas, H. J. Wörner, Opt. Express 25(22), 27506 (2017). 